\title{
Torsion of right middle lobe after a right upper lobectomy Chih-Hao Chen ${ }^{1}$, Tzu-Ti Hung ${ }^{1}$, Tung-Ying Chen ${ }^{2}$ and Hung-Chang Liu*1
}

Address: ${ }^{1}$ Department of Thoracic Surgery, Mackay Memorial Hospital, Taipei City, Taiwan and ${ }^{2}$ Department of Pathology, Mackay Memorial Hospital, Taipei City, Taiwan

Email: Chih-Hao Chen - musclenet2003@yahoo.com.tw; Tzu-Ti Hung - tzdi0921731610@yahoo.com.tw; TungYing Chen - wax921@yahoo.com.tw; Hung-Chang Liu* - cs520915@yahoo.com.tw

* Corresponding author

Published: 16 April 2009

Journal of Cardiothoracic Surgery 2009, 4:16 doi:10.1186/1749-8090-4-16
Received: 27 March 2009

Accepted: 16 April 2009

This article is available from: http://www.cardiothoracicsurgery.org/content/4/1/16

(c) 2009 Chen et al; licensee BioMed Central Ltd.

This is an Open Access article distributed under the terms of the Creative Commons Attribution License (http://creativecommons.org/licenses/by/2.0), which permits unrestricted use, distribution, and reproduction in any medium, provided the original work is properly cited.

\begin{abstract}
Lobar torsion after lung resection is a quite rare complication. A 50-year-old woman presented typical features on chest radiographs and CT(computed tomography) scan of lobar torsion after a right upper lobectomy. After emergency lobectomy of right middle lobe, the patient recovered well and discharged 10 days after the second operation.
\end{abstract}

\section{Background}

Resection of right upper lobe is a common procedure in patients with lung cancer. Infrequently, the fissure between the middle lobe and lower lobe is well developed so that torsion of the middle lobe might occur during or after an operation, especially when pneumopexy is not performed.

\section{Case presentation}

A 50-year-old woman presented 2-month cough and mild symptoms of hemoptysis. On routine chest radiograph of regular health examination, the patient was found to have a mass measuring around $3.5 \mathrm{~cm}$ in greatest diameterin right upper lung field (Figure 1A). Subsequent bronchoscopic brushing cytology confirmed the mass to be malignant. 7 days after admission, the patient underwent lobectomy and radical lymph node dissection through a standard right posterolateral thoracotomy. The chest radiograph on postoperative day 1 and 2 showed adequate lung expansion with no obvious abnormality. 5 days after operation, the patient presented fever and mild dyspnea. Follow-up chest radiograph showed a wedge-shaped opacity of large area in the middle lung field (Figure 1B). Bedside bronchoscopy showed tight orifice of right mid- dle lobe. CT scan showed collapse and hemorrhagic consolidation in right middle lobe (Figure 2). Under the impression of torsion, the patient underwent explorative thoracotomy. Under direct visualization, the right middle lobe was found to have a 720-degree counterclockwise torsion along the pedicle axis. An emergency lobectomy was performed. The patient was discharged 10 days after operation.

Lobar torsion can occurred in traumatic, spontaneous and postoperative conditions. Its occurrence is quite rare [1]. Prompt diagnosis of the torsion is quite difficult and must be differentiated from sputum impaction and lobar pneumonia. Because of the other 2 conditions may response well to chest physical therapy and effective antibiotic therapy respectively. The physical findings, including fever, tachycardia, dyspnea and decreased breath sounds, are not specific to make a diagnosis. The key steps are radiographic and bronchoscopic. Typical radiographic findings include homogenous consolidation in plain film and absence of contrast enhancement in the affected lobe on CT scan [2]. A careful bronchoscopic examination may reveal abnormally tight and obstructed orifice of the affected lobe. Right middle lobe is by far the most com- 


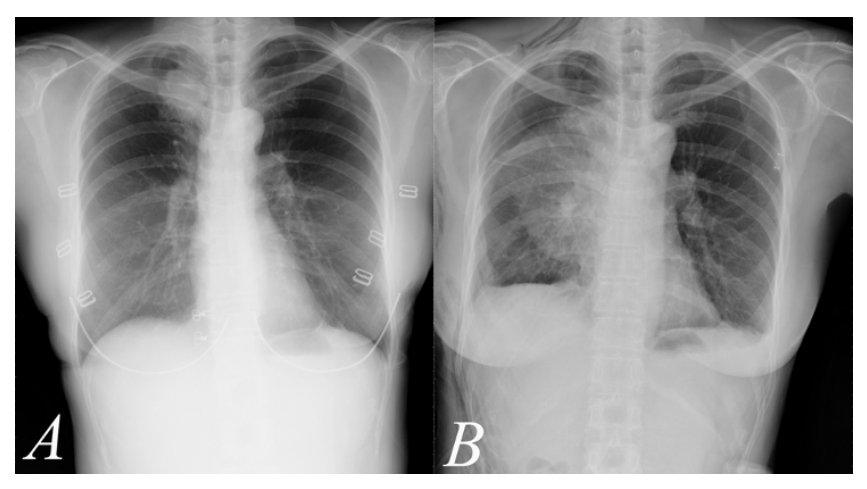

Figure I

A. mass measuring around $3.5 \mathrm{~cm}$ in greatest diameterin right upper lung field. B. Follow-up chest radiograph showed a wedge-shaped opacity of large area in the middle lung field.

mon afftected lobe. Pneumopexy is a key procedure to prevent such complication.

\section{Conclusion}

Pneumopexy with the use of sutures to anchor the middle lobe to the lower lobe after a right upper lobectomy must be practiced in all cases to reduce the risk of lobar torsion, especially if the fissure is well developed.

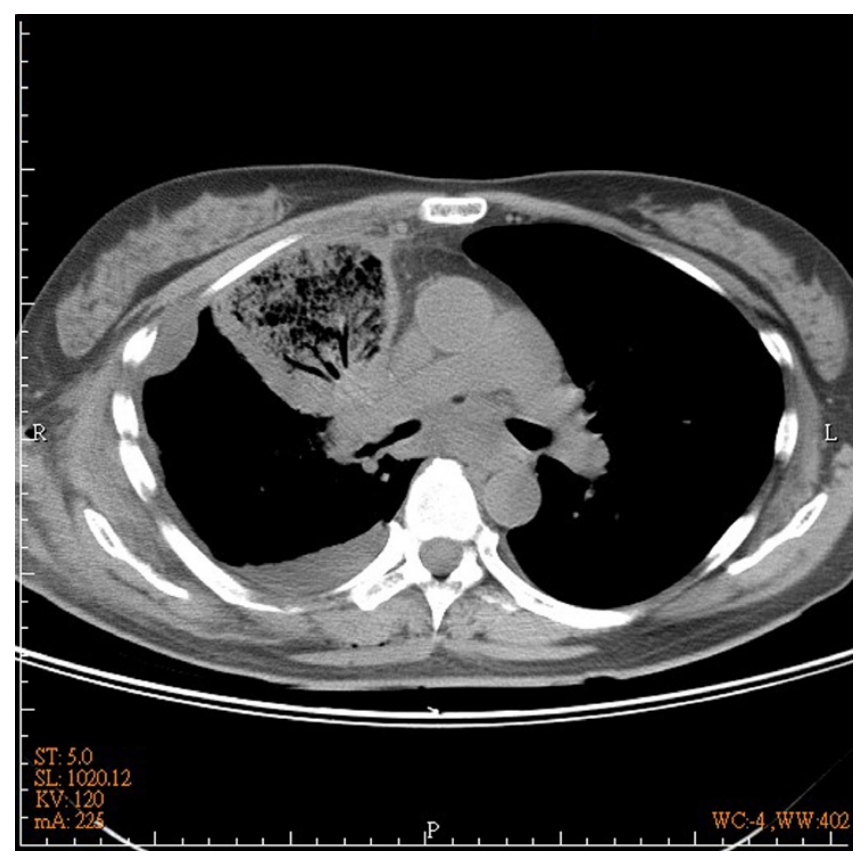

Figure 2

CT scan showed collapse and hemorrhagic consolidation in right middle lobe.

\section{Consent}

Written informed consent was obtained from the patient for publication of this case report and any accompanying images. A copy of the written consent is available for review by the Editor-in-Chief of this journal.

\section{Competing interests}

The authors declare that they have no competing interests.

\section{Authors' contributions}

CHC: the main author to write the article. TTH: collection of data and help us to revise the manuscript. TYC: help us for clinical pathological interpretation. HCL: final approval of the manuscript.

\section{References}

I. Cable DG, Deschamps C, Allen MS, Miller DL, Nichols FC, Trastek VF, Pairolero PC: Lobar torsion after pulmonary resection: presentation and outcome. J Thorac Cardiovasc Surg 200I, 122:109|-3.

2. Felson $B$ : Lung torsion: radiographic findings in nine cases. Radiology 1987, 162:631-8.
Publish with BioMed Central and every scientist can read your work free of charge

"BioMed Central will be the most significant development for disseminating the results of biomedical research in our lifetime. " Sir Paul Nurse, Cancer Research UK

Your research papers will be:

- available free of charge to the entire biomedical community

- peer reviewed and published immediately upon acceptance

- cited in PubMed and archived on PubMed Central

- yours - you keep the copyright
BioMedcentral 